\title{
Modelling HIVIAIDS Transmission Dynamics Considering Counselling, Vaccination and Antiretroviral Therapy (ART) in a Population of Varying Size
}

\author{
Udoo Iorlumun Joseph Martins ${ }^{1,}$, , Kimbir Richard Anande ${ }^{2}$, Remilekun Mathew Odekunle $^{3}$ \\ ${ }^{1}$ Department of Mathematics, School of Sciences, College of Education, Zing, Taraba State, Nigeria \\ ${ }^{2}$ Department of Mathematics, Statistics and Computer Science, Federal University of Agriculture, Makurdi, Benue State, Nigeria \\ ${ }^{3}$ Department of Mathematics, School of Pure and Applied Sciences, Modibbo Adama University of Technology, Yola, Adamawa State, Nigeria
}

Email address:

iorlumunudoo@gmail.com (I. J. M. Udoo), anandekimbir@yahoo.com (R. A. Kimbir), remiodekunle_@yahoo.com (R. M. Odekunle)

To cite this article:

Udoo Iorlumun Joseph Martins, Kimbir Richard Anande, Remilekun Mathew Odekunle. Modelling HIV/AIDS Transmission Dynamics Considering Counselling, Vaccination and Antiretroviral Therapy (ART) in a Population of Varying Size. American Journal of Applied Mathematics. Vol. 3, No. 6, 2015, pp. 271-282. doi: 10.11648/j.ajam.20150306.16

\begin{abstract}
A mathematical model of the transmission dynamics of HIV/AIDS, incorporating counselling, vaccination and antiretroviral therapy (ART) in a varying population, is presented. The existence and stability of the disease-free equilibrium states of the variants of the model are investigated, from which threshold values for vaccination and ART administration rates are established. Furthermore, numerical experiments are carried out to illustrate the effects of vaccination and ART, separately and in combination, on the transmission dynamics of HIV/AIDS in such populations.
\end{abstract}

Keywords: HIV/AIDS, Counselling, Vaccination, ART, Mathematical Model, Stability, Eradication, Threshold Values

\section{Introduction}

Despite all medical advances, infectious diseases still continue to pose a significant threat to the health of our society. They invade human populations and spread amongst its members, sometimes causing large number of fatalities, on recurrent basis. One such example that immediately comes to mind is the Acquired Immune Deficiency Syndrome (AIDS) pandemic, which is caused by an infectious agent known as the Human Immunodeficiency Virus (HIV), usually the type-1 retrovirus. Since the initial reporting of the AIDS pandemic in 1981 in the United States of America (USA) by the Centers for Disease Control and prevention [5], the worldwide spread of the pandemic has been far reaching. Global and regional estimates of HIV have been provided by the Joint United Nations Programme on HIV/AIDS (UNAIDS) and the World Health Organization (WHO) since the late 1980s and country specific estimates since 1996 [25, 8]. While the early 1980s AIDS cases were confined mostly to the homosexual men, hemophiliacs, and intravenous (IV) drug users in North America and Europe [5], today there is no geographical area, class, and cultural group of the world untouched by this pandemic [16]. It is common to the young and adults.
Individuals aged 15 years and above, are the most susceptible group [26]. This is because they are more sexually active.

The virus can be transmitted through sexual intercourse with an infected person, pregnancy (that is, from an infected mother to fetus), childbirth, breastfeeding, or exposure to contaminated needles/ blood products.HIV is associated with severe break down of the immune system of the affected person, rendering the body to be immune-deficient, resulting into catastrophic susceptibility of the infected person to opportunistic infections like Tuberculosis (TB), Pneumonia, meningitis, cancers and the gastrointestinal tract infections, which may result into illness and death [6, 21, 19].

The African Continent is the worst hit by the HIV pandemic, with Sub- Saharan Africa remaining the most severely affected, with nearly 1 in every 20 adults (4.9\%) living with HIV and accounting for $69 \%$ of the people living with HIV worldwide [27]. The impact of the epidemic has also badly affected households, schools, working places and economies $[24,2]$. After Sub-Saharan Africa, the regions most heavily affected are the Caribean and Eastern Europe and Central Asia, where $1.0 \%$ of adults were living with HIV in 2011 [27].

Since the start of the epidemic in 1981, about 75 million people have become infected with HIV. In 2012 alone, about 1.6 million people, died from AIDS - related causes 
worldwide [28]. Similarly, in the same year, surveillance data compiled by the Joint United Nations Programme on HIV/AIDS estimates that about 35.3 million people were living with HIV globally [28]; although the burden of the epidemic continues to vary considerably between countries and regions.

Although the number of people newly infected with HIV is declining worldwide, the number of people (adults and children) who acquired HIV infection in 2012 was about 2.3 million [28], lower than that of 2010, which stood at about 2.7 million people. The sharpest decline in the number of people acquiring HIV infection has occurred in the Caribean (42\%) and Sub-Saharan Africa (25\%). Despite these gains, SubSaharan Africa accounted for $71 \%$ of the adults and children newly infected in 2011 [27], underscoring the importance of continuing and strengthening prevention efforts in the region and other regions of the world.

Presently, there is no known medical cure for HIV infection nor is there a vaccine to prevent HIV infection. The most plausible HIV control measure involves prevention. These include condom use, abstinence, male circumcision and faithfulness. Condom use and male circumcision were shown to have a big impact on HIV with male circumcision alone reducing HIV transmission rate by $37 \%[30,20]$. Treatment of HIV consists of a combination of antiretroviral therapies (ARTs). ART blocks the replication of the virus and thus increases the life span of HIV-infected individuals. It does not lead to viral eradication within individuals and hence does not cure [29]. The effect of ART on HIV largely depends on the stage of infection at which treatment is initiated, levels of coverage, and the scale and stage of HIV epidemic that the community is experiencing. ART has been shown to have a big impact on HIV if the coverage is high [29].

Presently, extensive researches for the discovery of anti-HIV preventive and therapeutic vaccines are currently going on in different parts of the world. However, of the over 190 vaccine trials that have been completed to date against HIV/AIDS disease [7], only the results from the phase-3 clinical trials in Thailand of an anti-HIV preventive vaccine was released in late 2009 [26, 12], revealed $31.2 \%$ reduction in the risk of HIV infection, making this the first HIV vaccine to have a statistically significant effect. The results showed that this vaccine may be valuable in a community setting with largely heterosexual risk [12].

\section{Related Works}

Several mathematical models for the control and treatment of HIV/AIDS have been formulated and analyzed [18, 9, 14, $15,22,10,23]$. Similarly, despite the fact that no known vaccine against HIV infection exists, mathematical models for vaccination against HIV/AIDS have been formulated to predict the potential public and clinical impacts of such a vaccine $[4,17,13]$

To study the effects of counselling and antiretroviral therapy on the dynamics of HIV/AIDS in a homogeneously mixing population, [15] proposed and studied a one-sex model of the disease with proportionate recruitment into the population. Their one-sex model forms the motivation for this study which is intended to investigate the effects of counseling, vaccination and ART on HIV/AIDS transmission dynamics. This model extends the model by Kimbir and Oduwole [15] by incorporating the vaccination of susceptible individuals against HIV infection. Vaccination has been found to have induced permanent immunity against infectious diseases, like Measles, Smallpox, Rubella, to name but a few. Moreover, it is believed that a vaccine against HIV/AIDS, even one that is partially effective, could have a tremendous impact on the control of the infection in developing countries [11, 1].We therefore intend to investigate the potential community-wide effects of such a preventive vaccine (though not $100 \%$ perfect) in conjunction with counseling and ART.

This study is organized as follows. The model equations and the stability analyses of the different variants of the model are presented and carried out in sections 3 and 4, respectively. Some numerical examples are given in section 5 , and the discussions and conclusion in section 6 .

\section{Formulation of the Model}

We begin, for clarity, by presenting the model by [15].

Let $S(\mathrm{t})$ be the number of susceptible individuals at time $\mathrm{t}, \mathrm{I}(\mathrm{t})$ the number of infected individuals at time $\mathrm{t}, \mathrm{R}(\mathrm{t})$ the number of infected individuals receiving ART at time $t$, respectively. Then, using the flow diagram in Fig. 1 below, the one-sex model by [15] was given as follows:

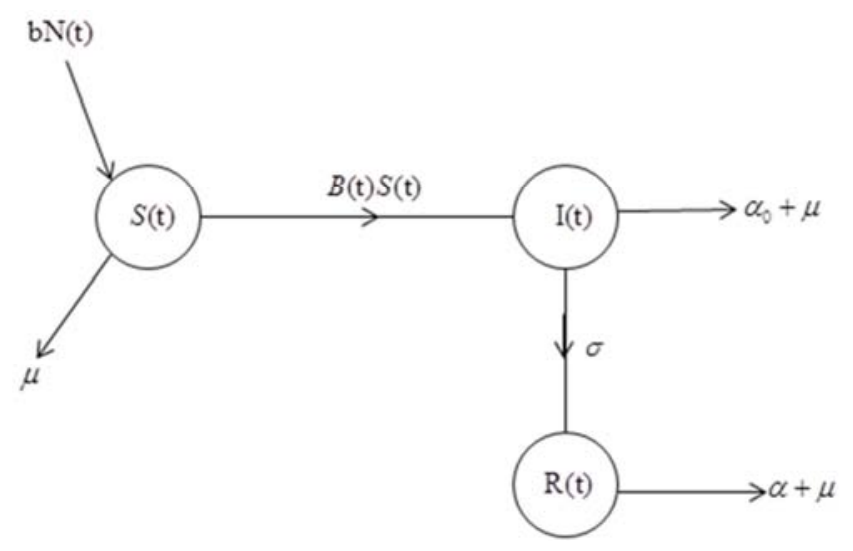

Fig. 1. Flow diagram of the Model by Kimbir and Oduwole [15].

$$
\left.\begin{array}{l}
\frac{d S}{d t}=b N(\mathrm{t})-\mathrm{B}(\mathrm{t}) \mathrm{S}(\mathrm{t})-\mu \mathrm{S}(\mathrm{t}) \\
\frac{d I}{d t}=B(\mathrm{t}) \mathrm{S}(\mathrm{t})-\left(\alpha_{0}+\mu+\sigma\right) I(\mathrm{t}) \\
\frac{d R}{d t}=\sigma I(\mathrm{t})-(\alpha+\mu) R(\mathrm{t})
\end{array}\right\},
$$

where

$$
N(\mathrm{t})=S(\mathrm{t})+\mathrm{I}(\mathrm{t})+\mathrm{R}(t)
$$

$b$ is the recruitment rate into the population, $\mu$ is the 
natural death rate, $\alpha_{0}$ is the death rate due to infection, $\alpha$ is the death rate of infectives receiving ART and $\sigma$ is the treatment rate. We assume that

$$
\alpha=\alpha_{0} e^{-k T}, \alpha<\alpha_{0}
$$

The incidence rate $\mathrm{B}(\mathrm{t})$ at time $\mathrm{t}$ is given as in Hsieh [10], by

$$
\mathrm{B}(\mathrm{t})=\frac{c \beta I+c^{\prime} \beta^{\prime} R}{N},
$$

where $\beta$ is the probability of transmission by an individual in class $I$ and $\beta^{\prime}$ is the probability of transmission by an individual in class $R, c$ and $c^{\prime}$ are, respectively, the average number of sexual partners per unit time for individuals in class $I$ and $R . \alpha_{0}, \alpha$ and $\sigma$ are as defined above. $T$ is the maximum lifespan after infection and $k$ is the efficacy of ART. $c \beta$ and $c^{\prime} \beta^{\prime}$ are the net transmission rates for the classes $I$ and $R$, respectively. As a result of counseling, it is assumed that $c^{\prime} \beta^{\prime}<c \beta$.

\subsection{The New Model Equations}

Using the flow diagram in Fig.2 below, the extended version of the model by [15] that incorporates the number of vaccinated susceptibles $\mathrm{V}(\mathrm{t})$ at time $\mathrm{t}$ is presented below. $\delta$ denotes the proportion of vaccinated susceptibles or the rate of vaccination per unit time.

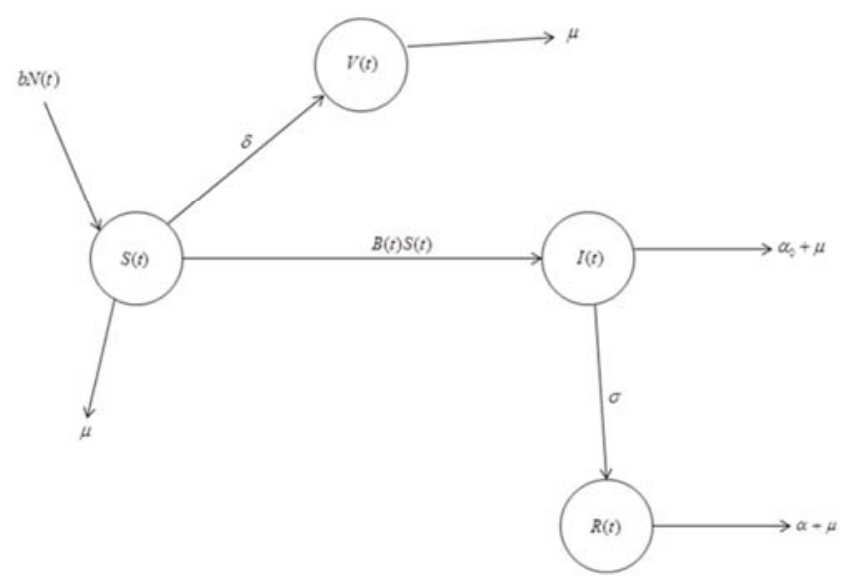

Fig. 2. Flow diagram of the extended Model.

$$
\begin{aligned}
& \frac{d S}{d t}=b N(\mathrm{t})-\mathrm{B}(\mathrm{t}) \mathrm{S}(\mathrm{t})-(\delta+\mu) \mathrm{S}(\mathrm{t}) \\
& \frac{d V}{d t}=\delta S(\mathrm{t})-\mu \mathrm{V}(\mathrm{t}) \\
& \frac{d I}{d t}=B(\mathrm{t}) S(\mathrm{t})-\left(\alpha_{0}+\mu+\sigma\right) \mathrm{I}(\mathrm{t}) \\
& \frac{d R}{d t}=\sigma I(\mathrm{t})-(\alpha+\mu) \mathrm{R}(\mathrm{t})
\end{aligned}
$$

where

$$
N(\mathrm{t})=S(\mathrm{t})+\mathrm{V}(\mathrm{t})+\mathrm{I}(\mathrm{t})+\mathrm{R}(\mathrm{t}) .
$$

Similarly,

$$
\mathrm{B}(\mathrm{t})=\frac{c \beta I+c^{\prime} \beta^{\prime} R}{N} .
$$

Finally, we assume that

$$
\alpha=\alpha_{0} e^{-k T}, \alpha<\alpha_{0} .
$$

Adding the equations in system (1) above, we get

$$
\frac{d N}{d t}=(b-\mu) N-\alpha_{0} I-\alpha R
$$

We consider the equations in proportions, as this reduces the number of equations. Furthermore, proportions of infected individuals define prevalence of infection, which has biological meaning.

Let

$$
s=\frac{S}{N}, v=\frac{V}{N}, i=\frac{I}{N}, r=\frac{R}{N},
$$

then, from the first equation in (6), we have

$$
\begin{aligned}
s^{\prime} & =\frac{1}{N}\left[S^{\prime}-s N^{\prime}\right] \\
& =\frac{1}{N}\left[b N-B S-(\delta+\mu) \mathrm{S}-s\left\{(\mathrm{~b}-\mu) \mathrm{N}-\alpha_{0} I-\alpha R\right\}\right] \\
& =b-\left(c \beta i+c^{\prime} \beta^{\prime} r\right) s-(b+\delta) s+\alpha_{0} i s+\alpha r s
\end{aligned}
$$

The remaining equations in proportions $i, v$, and $r$ are obtained in a similar way, namely:

$$
\left.\begin{array}{l}
i^{\prime}=\left(c \beta i+c^{\prime} \beta^{\prime} r\right) s-\left(b+\alpha_{0}+\sigma\right) i+\alpha_{0} i^{2}+\alpha r i \\
v^{\prime}=\delta s-b v+\alpha_{0} i v+\alpha r v \\
r^{\prime}=\sigma i-(b+\alpha) r+\alpha_{0} i r+\alpha r^{2}
\end{array}\right\} .
$$

Since

$$
s+i+v+r=1
$$

the governing model equations are

$$
\left.\begin{array}{l}
i^{\prime}=\left(c \beta i+c^{*} \beta^{*} r\right)(1-v-i-r)-\left(b+\alpha_{0}+\sigma\right) i+\alpha_{0} i^{2}+\alpha r i \\
v^{\prime}=\delta(1-c-i-r)-b v+\alpha_{0} i v+\alpha r v \\
r^{\prime}=\sigma i-(b+\alpha) r+\alpha_{0} i r+\alpha r^{2}
\end{array}\right\}
$$

\subsection{The DFE State of the Submodel Without an Intervention Measure (i.e., $\delta=\sigma=0$ )}

In the absence of vaccination and ART we have that 
$c^{*}=\beta^{*}=v=r=0$, and the model equations (9) reduce to the single model equation in $i$, namely

$$
i^{\prime}=c \beta i(1-i)-\left(b+\alpha_{0}\right) i+\alpha_{0} i^{2} .
$$

At equilibrium state $i^{\prime}=0$ and the two roots of equation (10) above are $i^{*}=0$, which corresponds to the disease-free equilibrium (DFE) state, and

$$
i^{*}=\frac{c \beta-\left(\mathrm{b}+\alpha_{0}\right)}{c \beta-\alpha_{0}}=\frac{\left(b+\alpha_{0}\right)\left(R_{0}-1\right)}{\left(b+\alpha_{0}\right) R_{0}-\alpha_{0}},
$$

which corresponds to an endemic equilibrium state, where

$$
R_{0}=\frac{c \beta}{\mu+\alpha_{0}} \text {. }
$$

$R_{0}$ is a threshold value that determines the persistence or otherwise of infection in the population.

Now, $0 \leq i<1$, therefore it is necessary that $R_{0}>1$. If $R_{0}=1$, then $i^{*}=0$ is the only equilibrium point, and is globally asymptotically stable (GAS) in $(0,1)$, otherwise there is exactly one positive equilibrium point

$$
i^{*}=\frac{c \beta-\left(\mathrm{b}+\alpha_{0}\right)}{c \beta-\alpha_{0}}=\frac{\left(b+\alpha_{0}\right)\left(R_{0}-1\right)}{\left(b+\alpha_{0}\right) R_{0}-\alpha_{0}}
$$

which is GAS, as shown in [15].

\subsection{The DFE State of the Submodel with Only Susceptible \\ Individuals Vaccinated Against HIV Infection (i.e., $\delta>0$, $\sigma=0)$}

Here, we consider the situation when only susceptible individuals are vaccinated against HIV infection. In this case, $c^{*}=\beta^{*}=r=0$ and the model equations (9) reduce to

$$
\left.\begin{array}{l}
i^{\prime}=c \beta i(1-v-i)-\left(b+\alpha_{0}\right) i+\alpha_{0} i^{2} \\
v^{\prime}=\delta(1-v-i)-b v+\alpha_{0} i v
\end{array}\right\}
$$

where with $\delta=0$, we recover equation (10).

At the equilibrium state, $i^{\prime}=v^{\prime}=0$, and the DFE state for the above submodel is given as

$$
E_{0}=\left(\begin{array}{c}
-- \\
i, v
\end{array}\right)=\left(0, \frac{\delta}{b+\delta}\right) \text {. }
$$

Define the region

$$
E=\left\{(i, v) \in R_{+}^{2}: i>0, v \geq 0, i+v=1\right\} .
$$

$E$ is a triangular region as shown in Figure 3 below.

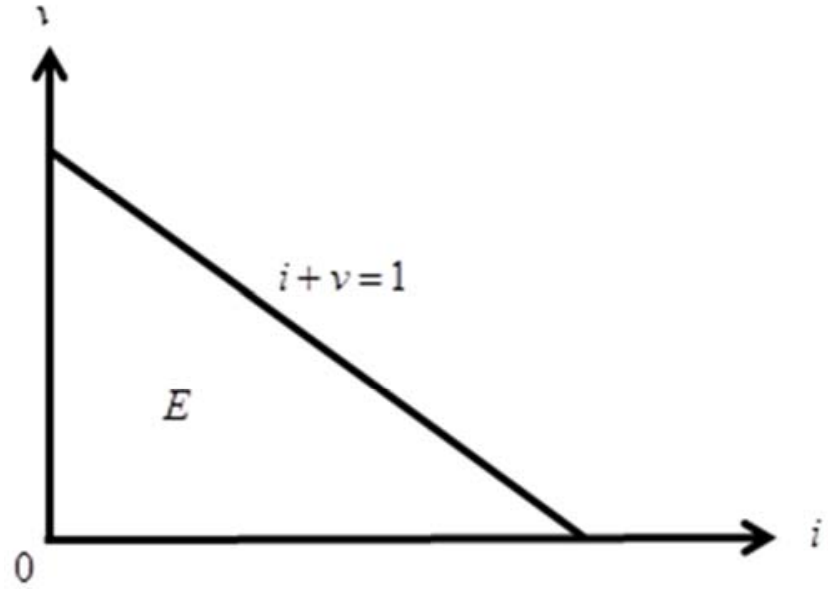

Fig. 3. A triangular region.

The Jacobian matrix evaluated at the DFE state $E_{0}$ is given by

$$
\begin{aligned}
& J_{\left(E_{0}\right)}=\left[\begin{array}{cc}
c \beta\left(\frac{b}{b+\delta}\right)-\left(b+\alpha_{0}\right) & 0 \\
\frac{\alpha_{0} \delta}{b+\delta}-\delta & -(b+\delta)
\end{array}\right] . \\
& \operatorname{tr} J_{\left(\mathrm{E}_{0}\right)}=\left(b+\alpha_{0}\right)\left(R_{0 v}-1\right)-(b+\delta)<0
\end{aligned}
$$

Provided

$$
R_{0 v}<1
$$

where $R_{o v}=\frac{c \beta}{\left(\mu+\alpha_{0}\right)} \cdot \frac{b}{(\mu+\delta)}$,

and,

$$
\operatorname{det} J_{\left(\mathrm{E}_{0}\right)}=\left(b+\alpha_{0}\right)(b+\delta)-c \beta b>0,
$$

provided that the condition in (14) above holds.

Therefore, $E_{0}$ is LAS. Thus, we have proved the following theorem.

Theorem 3.3.1

Given that $b, \alpha_{0}, \delta, c \beta>0$. If $b+\delta>\alpha_{0} \quad$ and $0<R_{0 v}=\frac{c \beta b}{\left(b+\alpha_{0}\right)(b+\delta)}<1$, then there is a DFE state $\left(0, \frac{\delta}{b+\delta}\right)$ for the submodel (13) which Is locally asymptotically stable in the region $E$; otherwise there is a unique endemic equilibrium state $\left(i^{*}, v^{*}\right)$ which is locally asymptotically stable (LAS) in $E-\left\{\left(0, \frac{\delta}{b+\delta}\right)\right\}$.

Now, we shall show, firstly, that $E$ is invariant. We do this 
by showing, as in Beltrami [3], that the inner product of the vector field (defined by system (13)) with the inward normal to $E$ is non-negative.

From Fig.3 above, we see that the inward normal to the $i$-axis is $(0,1)$. Therefore, $(0,1)\left(i^{\prime}, \mathrm{v}^{\prime}\right)=\delta(1-i)>0$ (since $v=0$ on this axis and $i<1$ ).

Next, the inward normal to the $v$-axis is $(1,0)$, so that $(1,0)\left(i^{\prime}, \mathrm{v}^{\prime}\right)=0($ since $i=0$ on this axis).

Finally, on the line $i+v=1$, the inward normal is $(-1,-1)$, and we have $(-1,-1)\left(i^{\prime}, v^{\prime}\right)>0$, using similar arguments.

Thus, we have proved that $E$ is invariant.

To prove that there are no periodic solutions in $E$, we apply the Bendixon-Dulac criterion as in [10].

Let $h=\frac{1}{i v} ; f_{1}$ and $f_{2}$ be the right-hand sides of system (13), respectively. Then we have

$$
\frac{\partial}{\partial i}\left(h f_{1}\right)+\frac{\partial}{\partial v}\left(h f_{2}\right)=\frac{\alpha_{0}-c \beta}{v}+\frac{\delta}{i v^{2}}(i-1)<0
$$

(since $c \beta>b+\alpha_{0}>\alpha_{0}$ and $i<1$ ). Therefore, there are no periodic solutions in $E$.

This completes the proof of the theorem.

\subsection{The Submodel with Only Infected Individuals Receiving $\operatorname{ART}(\delta=0, \sigma>0)$}

In this case, $\delta=v=0$ and the normalized system of model equations $(9)$ reduces to

$$
\left.\begin{array}{l}
i^{\prime}=\left(c \beta i+c^{*} \beta^{*} r\right)(1-i-r)-\left(b+\alpha_{0}+\sigma\right) i+\alpha i r+\alpha_{0} i^{2} \\
\mathrm{r}^{\prime}=\sigma i-(b+\alpha) r+\alpha_{0} i r+\alpha r^{2}
\end{array}\right\} .
$$

If $\sigma=0$, we recover equation (10) in sub-section 3.1.1.

At equilibrium state, $i^{\prime}=r^{\prime}=0$, and the DFE state for this submodel is given as $E_{1}=(0,0)$.

Define the region

$$
D=\left\{(i, \mathrm{r}) \in R_{+}^{2}: i \geq 0, \mathrm{r} \geq 0, i+r=1\right\} .
$$

We notice that the system (15) recovers equations (8)-(9) of the submodel in Kimbir and Oduwole [15], with the following corresponding results, stated in the theorem below.

Theorem 3.4.1

Given that $b, \alpha_{0}, \alpha, c \beta, c^{\prime} \beta^{\prime}>0$. If $b+\alpha>\alpha_{0}$ and $0<R=\frac{c \beta}{b+\alpha_{0}+\sigma}<1$, then there is a DFE state $(0,0)$ in $D$

Which is locally and asymptotically stable (LAS), otherwise there exists an endemic state $\left(i^{*}, r^{*}\right)$, which is LAS in $D-\{(0,0)\}$.

\subsection{The General Model (i.e., $\delta>0, \sigma=0$ )}

This is the normalized system of model equations (9) above. That is

$$
\left.\begin{array}{l}
i^{\prime}=\left(c \beta i+c^{*} \beta^{*} r\right)(1-v-i-r)-\left(b+\alpha_{0}+\sigma\right) i+\alpha_{0} i^{2}+\alpha r i \\
v^{\prime}=\delta(1-c-i-r)-b v+\alpha_{0} i v+\alpha r v \\
r^{\prime}=\sigma i-(b+\alpha) r+\alpha_{0} i r+\alpha r^{2}
\end{array}\right\}
$$

At equilibrium state, $i^{\prime}=v^{\prime}=r^{\prime}=0$, and the DFE state for the general model is given as $E_{2}=\left(i^{*}, v^{*}, r^{*}\right)=\left(0, \frac{\delta}{b+\delta}, 0\right)$. The Jacobian matrix evaluated at $E_{2}$ is given by

$$
J_{\left(E_{2}\right)}=\left[\begin{array}{ccc}
c \beta\left(\frac{b}{b+\delta}\right)-\left(b+\alpha_{0}+\sigma\right) & 0 & c^{\prime} \beta^{\prime}\left(\frac{b}{b+\delta}\right) \\
-\delta+\frac{\alpha_{0} \delta}{b+\delta} & -(b+\delta) & -\delta+\frac{\alpha \delta}{b+\delta} \\
\sigma & 0 & -(b+\alpha)
\end{array}\right] .
$$

If $\delta=0$ or $\sigma=0$ we retrieve the Jacobian matrices in sub-sections 3.3.

The characteristic polynomial associated with the above matrix is

$$
P(\lambda)=\lambda^{3}+A \lambda^{2}+B \lambda+C=0
$$

where

$$
\begin{gathered}
A=\left(b+\alpha_{0}+\sigma\right)-c \beta\left(\frac{b}{b+\delta}\right)+(b+\delta)+(b+\alpha), \\
B=-(b+\delta)\left\{c \beta\left(\frac{b}{b+\delta}\right)-\left(b+\alpha_{0}+\sigma\right)\right\} \\
-(b+\alpha)\left\{c \beta\left(\frac{b}{b+\delta}\right)-\left(b+\alpha_{0}+\sigma\right)\right\}-c^{\prime} \beta^{\prime}\left(\frac{b}{b+\delta}\right) \sigma \\
+(b+\delta)+(b+\alpha),
\end{gathered}
$$

and

$$
\begin{aligned}
& C=\left\{\left(b+\alpha_{0}+\sigma\right)-c \beta\left(\frac{b}{b+\delta}\right)\right\}(b+\delta)(b+\alpha) \\
& -(b+\delta) c^{\prime} \beta^{\prime}\left(\frac{b}{b+\delta}\right) \sigma
\end{aligned}
$$

Now,

$$
A=\left(b+\alpha_{0}+\sigma\right)-c \beta\left(\frac{b}{b+\delta}\right)+(b+\delta)+(b+\alpha)>0
$$


provided that

$$
\begin{gathered}
c \beta b<(b+\delta)\left[\left(b+\alpha_{0}+\sigma\right)+(b+\delta)+(b+\alpha)\right] ; \\
B=-(b+\delta)\left\{c \beta\left(\frac{b}{b+\delta}\right)-\left(b+\alpha_{0}+\sigma\right)\right\} \\
-(b+\alpha)\left\{c \beta\left(\frac{b}{b+\delta}\right)-\left(b+\alpha_{0}+\sigma\right)\right\}-c^{\prime} \beta^{\prime}\left(\frac{b}{b+\delta}\right) \sigma \\
+(b+\delta)+(b+\alpha)>0
\end{gathered}
$$

provided that

$$
\begin{gathered}
\quad\{(b+\delta)+(b+\alpha)\} c \beta b+c^{\prime} \beta^{\prime} b \sigma \\
<\left(b+\alpha_{0}+\sigma\right)\left\{(b+\delta)^{2}+(b+\delta)(b+\alpha)\right\} \\
+(b+\delta)[(b+\delta)+(b+\alpha)] .
\end{gathered}
$$

and

$$
\begin{aligned}
& C=\left\{\left(b+\alpha_{0}+\sigma\right)-c \beta\left(\frac{b}{b+\delta}\right)\right\}(b+\delta)(b+\alpha) \\
& -(b+\delta) c^{\prime} \beta^{\prime}\left(\frac{b}{b+\delta}\right) \sigma>0
\end{aligned}
$$

provided that

$$
c \beta b(b+\alpha)+c^{\prime} \beta^{\prime} \sigma<\left(b+\alpha_{0}+\sigma\right)(b+\delta)(b+\alpha) .
$$

Also,

$$
\begin{gathered}
A B-C=\left[\left(b+\alpha_{0}+\sigma\right)-c \beta\left(\frac{b}{b+\delta}\right)+(b+\delta)+(b+\alpha)\right] \\
\times\left[\begin{array}{l}
-(b+\delta)\left\{c \beta\left(\frac{b}{b+\delta}\right)-\left(b+\alpha_{0}+\sigma\right)\right\} \\
-(b+\alpha)\left\{c \beta\left(\frac{b}{b+\delta}\right)-\left(b+\alpha_{0}+\sigma\right)\right\}
\end{array}\right. \\
-\left[\left\{\begin{array}{l}
\left\{\left(b+\alpha_{0}+\sigma\right)-c \beta\left(\frac{b}{b+\delta}\right)\right\}(b+\delta)(b+\alpha) \\
-(b+\delta) c^{\prime} \beta^{\prime}\left(\frac{b}{b+\delta}\right) \sigma
\end{array}\right]>0\right.
\end{gathered}
$$

provided that the conditions expressed in (17), (18),and (19) hold.

We observe from (19) that

$$
R_{v t}=\frac{c \beta b}{\left(b+\alpha_{0}+\sigma\right)(b+\delta)}+\frac{c^{\prime} \beta^{\prime} \sigma b}{(b+\alpha)\left(b+\alpha_{0}+\sigma\right)(b+\delta)} .
$$

$R_{v t}$ is the threshold parameter that determines the persistence or otherwise of HIV infection in the presence of vaccination and ART in the population.

Thus, the Routh-Hurwitz conditions (i) $A, B, C>0$ and (ii) $A B-C>0$ are satisfied. Hence, the roots of (16) have negative real parts, and the DFE is LAS.

Thus we have proved the following theorem.

Theorem 3.5.1

\section{Givethat}

$c \beta>0, c^{\prime} \beta^{\prime} \geq 0, c^{\prime} \beta^{\prime}<b+\alpha<c \beta, \delta>0, \sigma>0$

$R_{v t}<1,1>\delta>0,1>\sigma>0$, then there exists a unique DFE state $\left(0, \frac{\delta}{b+\delta}, 0\right)$ for the system (9) which is LAS.

\section{Suspension of Vaccination and ART (i.e., $\delta=\sigma=0$ )}

Let us assume that we begin with a non-zero proportion of individuals in the classes $V$ and $R$ as in the model (9) above then suspend vaccination of susceptible individuals against HIV infection and infecteds receiving ART. The model becomes

$$
\left.\begin{array}{l}
i^{\prime}=\left(c \beta i+c^{*} \beta^{*} r\right)(1-i-v-r)-\left(b+\alpha_{0}\right) i+\alpha_{0} i^{2}+\alpha r i \\
v^{\prime}=-b v+\alpha_{0} i v+\alpha r v \\
r^{\prime}=-(b+\alpha) r+\alpha_{0} i r+\alpha r^{2}
\end{array}\right\}
$$

and the DFE state for system $(21)$ is $(0,0,0)$. The Jacobian matrix evaluated at the DFE state is given by

$$
J_{(0)}=\left[\begin{array}{ccc}
c \beta-\left(b+\alpha_{0}\right) & 0 & c^{\prime} \beta^{\prime} \\
0 & -b & 0 \\
0 & 0 & -(b+\alpha)
\end{array}\right] .
$$

The characteristic polynomial of $J_{(0)}$, given by

$$
\left|J_{(0)}-\lambda I\right|=0
$$

has three roots, namely, $\lambda_{1}=c \beta-\left(\mathrm{b}+\alpha_{0}\right), \lambda_{2}=-b<0$, and $\lambda_{3}=-(\mathrm{b}+\alpha)<0$, respectively.

The DFE state $(0,0$,$) of system (21) is LAS if, and only if,$ $\lambda_{1}=c \beta-\left(\mathrm{b}+\alpha_{0}\right)<0$; that is, when

$$
R_{0}=\frac{c \beta}{b+\alpha_{0}}<1 .
$$

Therefore, we have proved the following theorem.

Theorem 4.1

Suppose we suspend vaccination of susceptibles against $H I V$ infection and ART for the infected individuals (i.e., $\delta=\sigma=0)$.If $R_{0}<1$, then the DFE state $(0,0,0)$ for 
the system (23) is LAS.

\section{Numerical Simulations}

In this section, we carry out some numerical simulations of the equations of the model (9), using hypothetical data.

Below is Table1, of parameter values for the numerical experiments.

Table 1. Table of Parameter Values for the Numerical Experiments.

\begin{tabular}{|c|c|c|c|c|c|c|c|c|c|c|c|c|c|c|}
\hline Fig. & Table & $b$ & $\alpha_{0}$ & $k$ & $T$ & $c$ & $c^{\prime}$ & $\boldsymbol{\beta}$ & $\boldsymbol{\beta}^{\prime}$ & $\delta$ & $\sigma$ & $i(0)$ & $\boldsymbol{v}(0)$ & $\boldsymbol{r}(0)$ \\
\hline 4 & A1 & 0.5 & 0.2 & 0 & 10 & 4 & 0 & 0.35 & 0 & 0 & 0 & 0.35 & 0 & 0 \\
\hline 5 & A2 & 0.5 & 0.2 & 0.1 & 10 & 4 & 3 & 0.35 & 0.25 & 0.15 & 0.3 & 0.35 & 0 & 0 \\
\hline 6 & A3 & 0.5 & 0.2 & 1 & 10 & 2 & 1 & 0.1 & 0 & 0.7 & 0.8 & 0.35 & 0 & 0 \\
\hline 7 & A4 & 0.5 & 0.2 & 0 & 10 & 2 & 0 & 0.1 & 0 & 0.7 & 0 & 0.35 & 0 & 0 \\
\hline 8 & A5 & 0.5 & 0.2 & 1 & 10 & 2 & 1 & 0.1 & 0 & 0 & 0.8 & 0.35 & 0 & 0 \\
\hline 9 & A6 & 0.5 & 0.2 & 0 & 10 & 2 & 0 & 0.1 & 0 & 0 & 0 & 0.35 & 0 & 0 \\
\hline
\end{tabular}

As shown in Table 1 above, the numerical experiments are meant to study the following situations:

1. Prevalence of HIV infection in the absence of any intervention measure (see Fig.4).

2. Prevalence of HIV infection when vaccination and ART rates are very low, in the presence of ineffective counselling (see Fig. 5).

3. Prevalence of HIV infection when vaccination and ART rates are very high, in the presence of very effective counselling (see Fig. 6).

4. Prevalence of HIV infection when ART is withdrawn but with very high vaccination rate, in the presence of very effective counselling (see Fig.7).

5. Prevalence of HIV infection when vaccination is withdrawn but with very high ART rates, in the presence very effective counselling (see Fig. 8).

6. Prevalence of HIV infection when vaccination and ART are suspended in the presence of very effective counselling (see Fig. 9).

Using the computer software Matlab, the fourth-order Runge-Kutta Integration scheme is adopted for our numerical simulations/experiments. The figures generated from the various parameter values in Table 1 are given below.

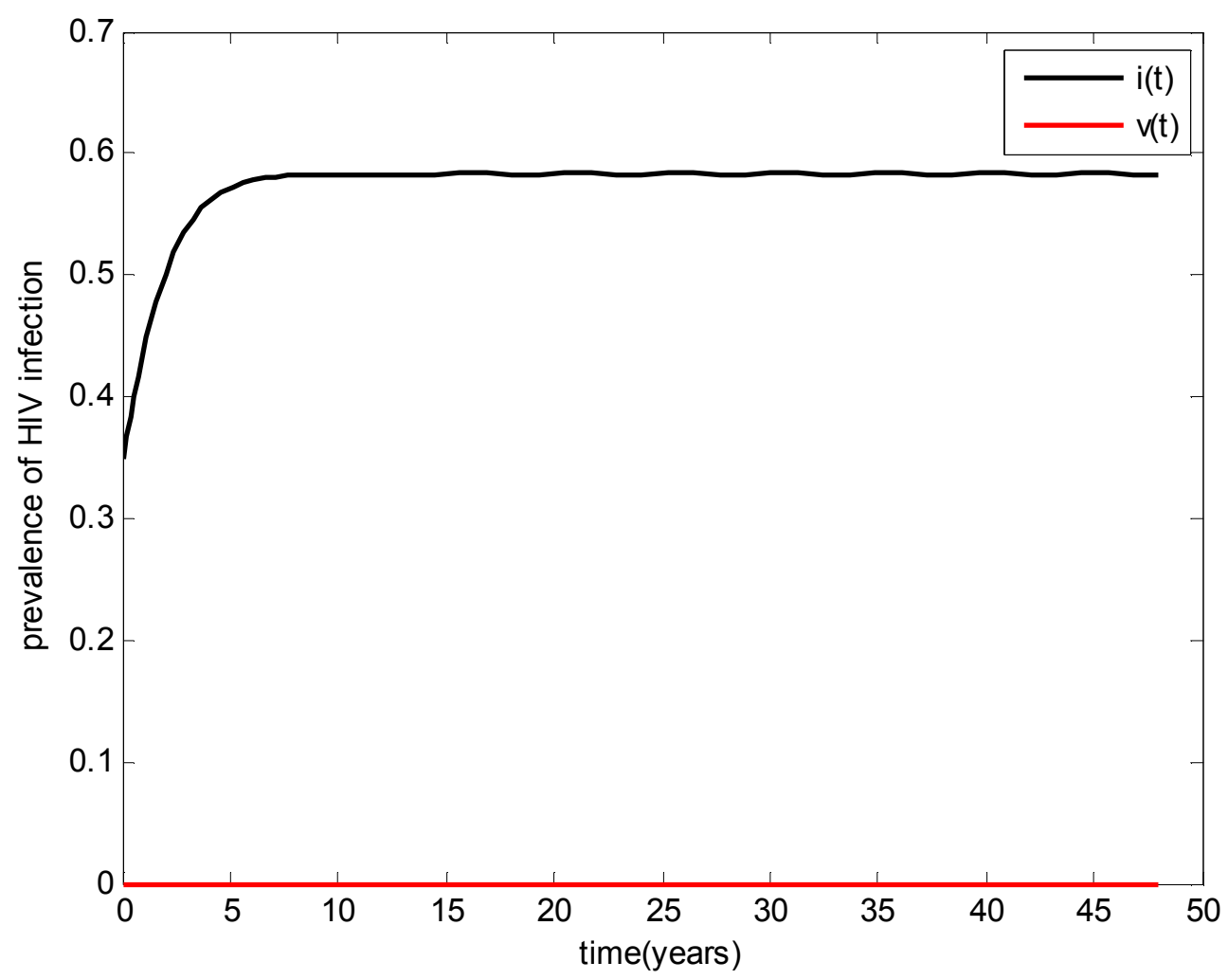

Fig. 4. Prevalence of infection without any intervention measure (i.e, $\delta=\sigma=0$ ).

Other parameter values are as given in Table 1 (A1).

Figure 4 shows an increasing prevalence of HIV infection in the absence of any intervention measure. 


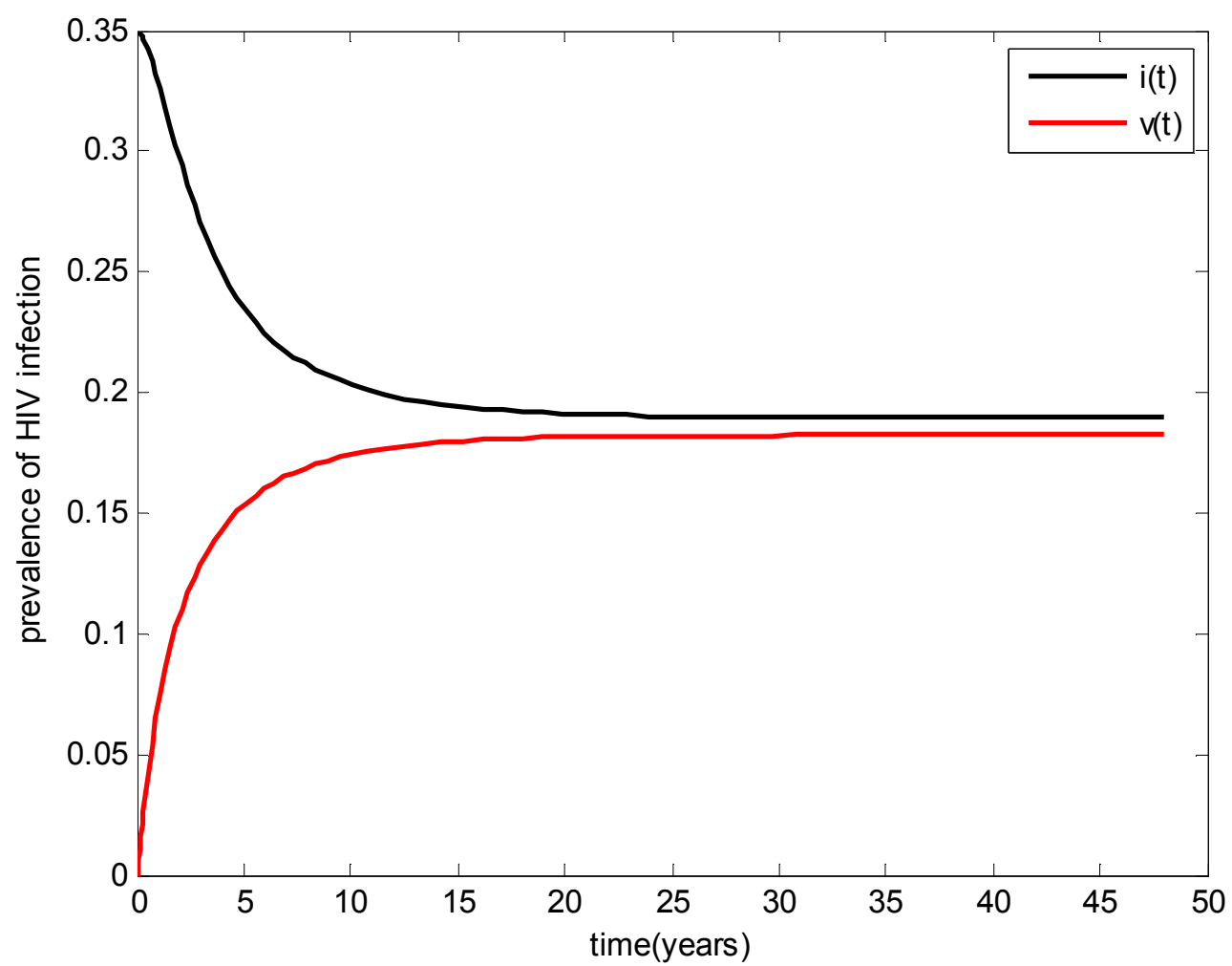

Fig. 5. Prevalence of HIV infection when the vaccination and ART rates are very low (i.e, $\delta=0.15, \sigma=0.30)$, in the presence of ineffective counselling.

Other parameter values are as given in Table 1 (A2).

Figure 5 shows an appreciable decline in prevalence of HIV infection when vaccination and ART rates are very low (i.e, $\delta=0.15, \sigma=0.30$ ).

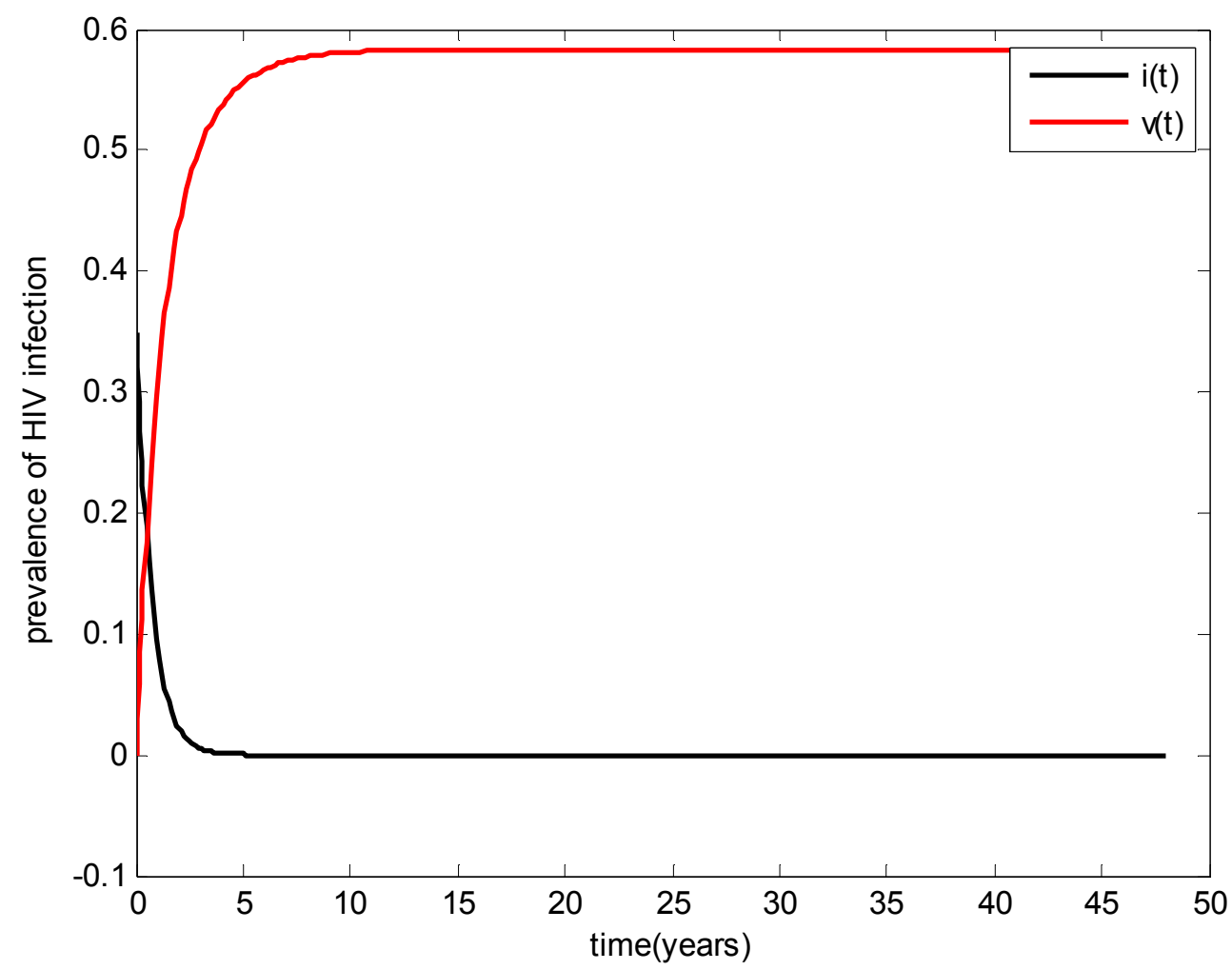

Fig. 6. Prevalence of HIV infection when the vaccination and ART rates are very high (i.e, $\delta=0.7, \sigma=0.8)$. 
Other parameter values are as given in Table 1 (A3).

Figure 6 depicts a very sharp decline in the prevalence of HIV infection when the vaccination and ART rates are very high in the presence of very effective counselling. This leads to the eradication of the disease in, about, less than 5 years time.

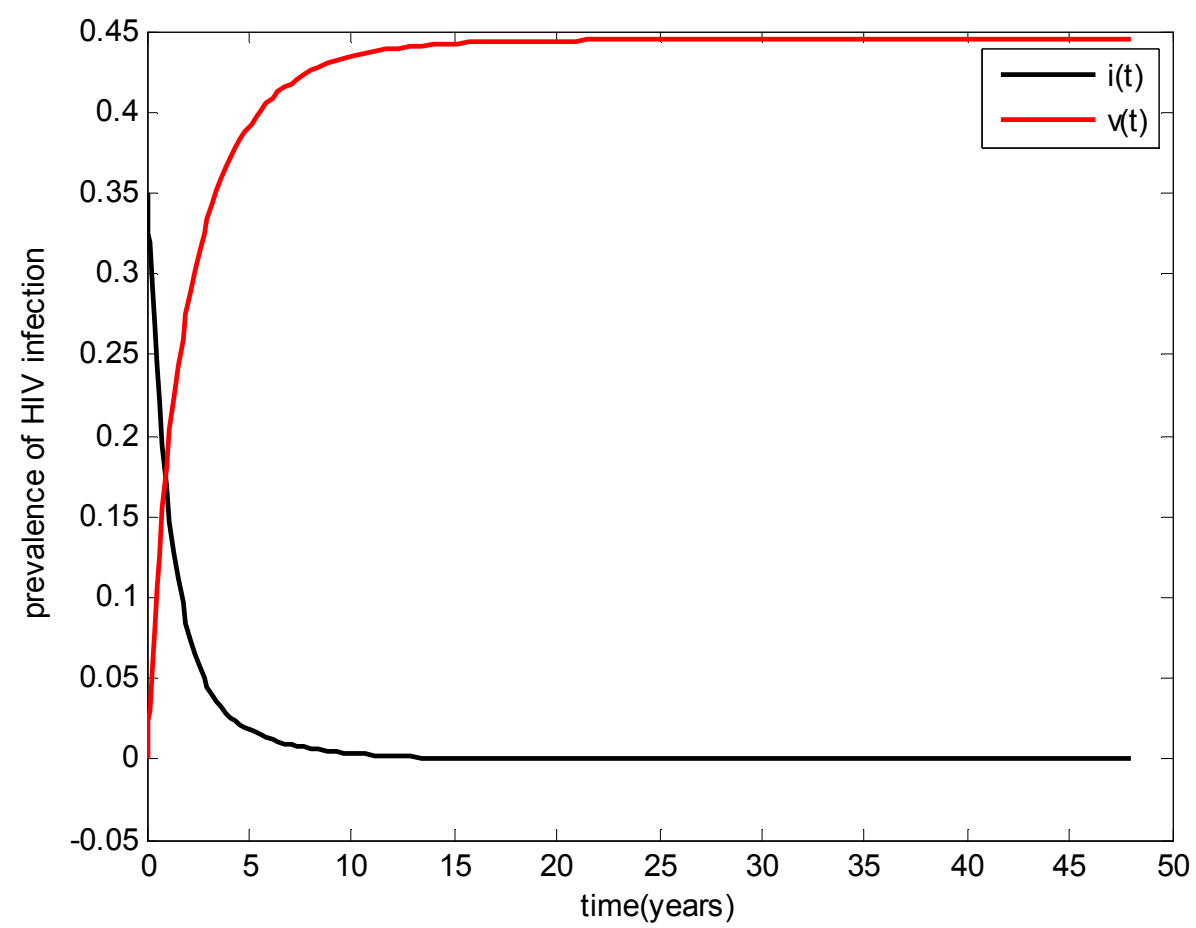

Fig. 7. Prevalence of HIV infection when ART is withdrawn but with very high vaccination rate, (i.e, $\delta=.7, \sigma=0)$, in the presence of very effective counselling.

Other parameter values are as given in Table 1 (A4).

Figure 7 shows a significant decline in the prevalence of HIV infection when ART is withdrawn but with very high vaccination rate in the presence of very effective counseling. This leads to the eradication of the disease in (about) 12years time.

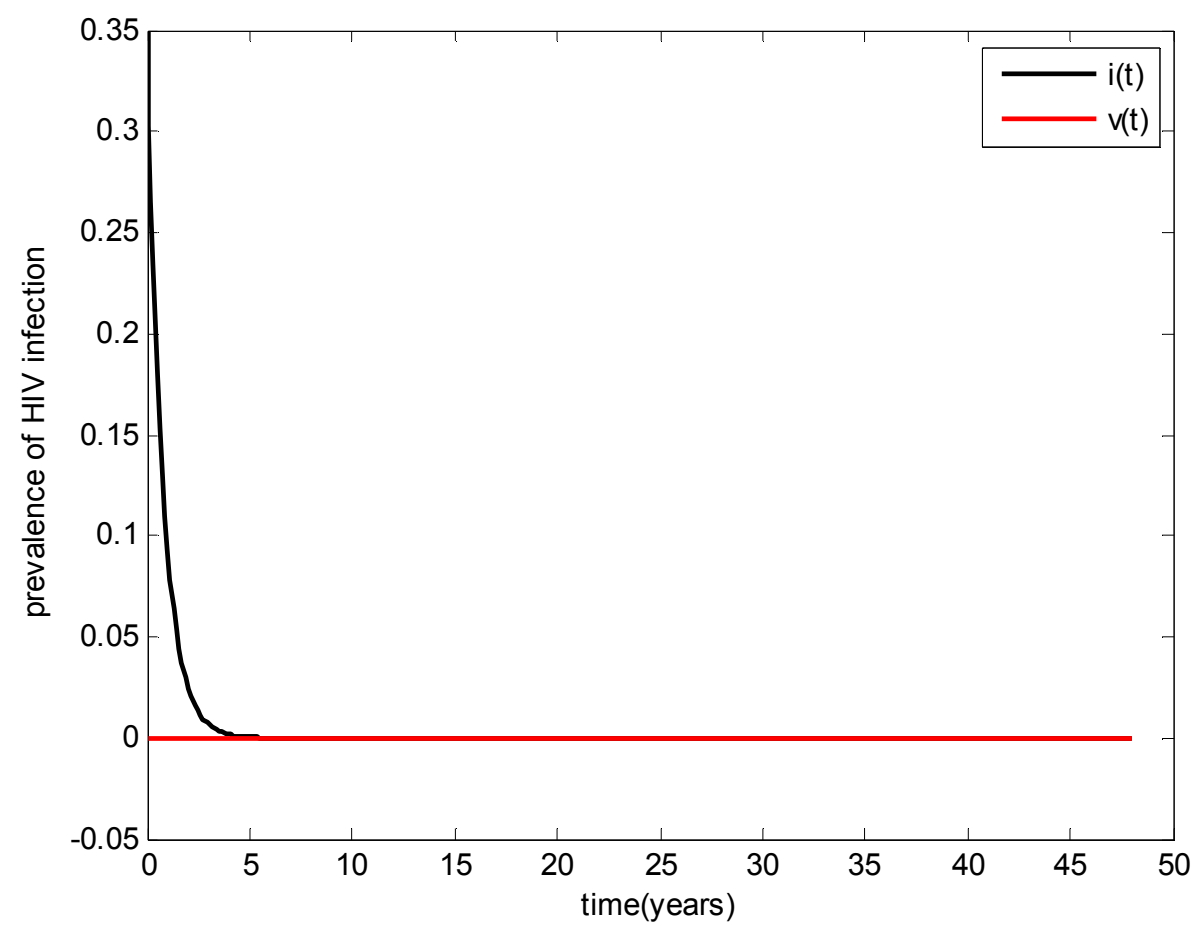

Fig. 8. Prevalence of HIV infection when we withdraw vaccination but with very high ART rate (i.e, $\delta=0, \sigma=0.8)$, in the presence of very effective counselling. 
Other parameter values are as given in Table 1(A5).

Figure 8 shows a very high decline in the prevalence of HIV infection when vaccination is withdrawn but with very high ART rate in the presence of very effective counselling. This leads to the eradication of the disease in (about) 6 years time. This is due to high efficacy level of ART (i.e, $k=1, \sigma=0.8$ ), and a sufficient reduction in number of sexual partners.

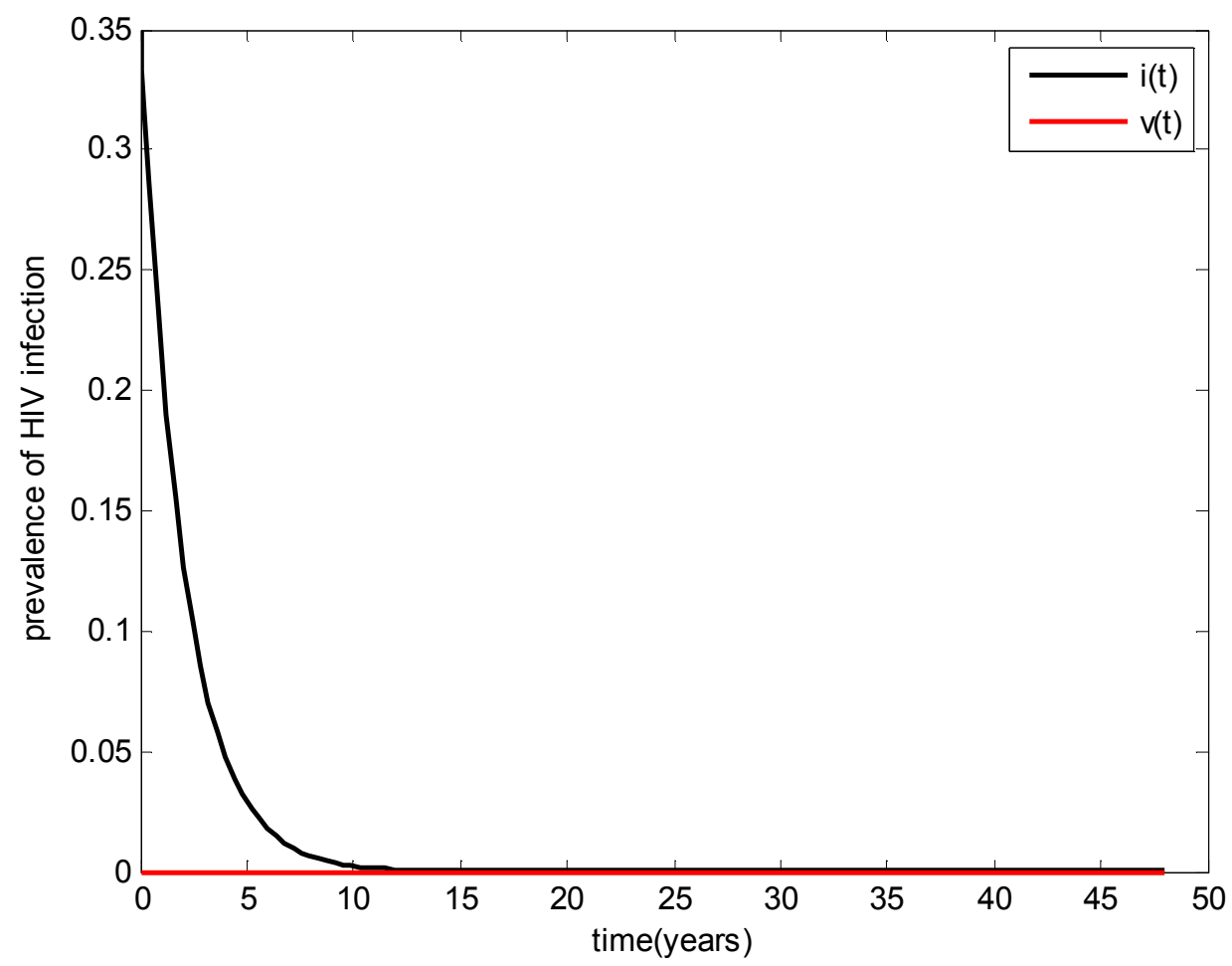

Fig. 9. Prevalence of HIV infection when we suspend vaccination and $A R T($ i. e, $\delta=0, \sigma=0)$, in the presence of very effective counselling.

Other parameter values are as given in Table 1 (A6).

Figure 9 shows the prevalence of HIV infection when we suspend vaccination and ART in the presence of very effective counselling. This leads to the eradication of the disease in (about) 10years time. This is as a result very small number of sexual partners.

Hence, this study confirms that counselling, vaccination and ART could be useful strategies for the control and eradication of HIV/AIDS from the population.

\section{Discussions and Conclusion}

An extended version of a one-sex deterministic mathematical model of HIV/AIDS transmission dynamics that incorporates vaccination of susceptible individuals against HIV infection with counselling and ART in a population of varying size has been proposed and studied. Threshold conditions, in terms of the model parameters, for the existence and stability of DFE states have been obtained for different variants of the model. The stability analysis of the DFES has been carried out using the linearization method and the Routh-Hurwitz stability criterion.

For the submodel without an intervention measure (that is, section 3.2), we note that, when the threshold parameter that determines the persistence or otherwise of infection, in the absence of an intervention measure, is less than unity, that is,
$0<R_{0}=\frac{c \beta}{\left(b+\alpha_{0}\right)}<1$, the disease dies out, hence, in this case, vaccination and /or ART are not necessary. If, however, $R_{0}>1$, then the disease establishes itself in the population and so becomes endemic.

For the submodel with only susceptibles vaccinated against HIV infection (that is, section 3.3), we note in Theorem 3.3.1 that, when the threshold parameter that determines the persistence or otherwise of infection, in the presence of a vaccination programme, is less than unity, that is, $0<R_{0 v}=\frac{c \beta b}{\left(b+\alpha_{0}\right)(b+\delta)}<1$, the DFES is LAS; otherwise there exists an endemic equilibrium state which is LAS. In this case, other alternative intervention measures, like ART, are necessary. And from the expression for $R_{0 v}<1$, the minimum proportion $\delta$ of susceptible individuals to be vaccinated for possible control and eradication of HIV is $\frac{c \beta b-b\left(b+\alpha_{0}\right)}{\left(b+\alpha_{0}\right)}$.

In section 3.4, the submodel with only infectives receiving ART, we recover the model by [15]. Results from their stability analysis revealed that, for an effective ART programme, it may be necessary to reduce the transmission probability and the average number of sexual partners of the 
infected individuals. These could be done through counseling and education; and from the expression for $R=\frac{c \beta}{b+\alpha_{0}+\sigma}<1$ the minimum proportion $\sigma$ of infected individuals to receive ART for possible control and eradication of HIV is $c \beta-\left(\mathrm{b}+\alpha_{0}\right)$.

Similar results are obtained in section 3.5 , that is, when vaccination and ART are administered jointly.

It is observed in theorem 4.1, that is, in section 4 , that even when we suspend the administration of vaccination and ART, it is still possible to control and eradicate HIV/AIDS from the population, provided that the intensity of disease transmission is sufficiently reduced through a very effective counseling programme.

Similarly, numerical results show that effective counseling and very high vaccination and ART coverage could bring about faster control and eradication of the disease from the population than it was the case with the model by [15], where counselling and ART were the only strategies employed (see Figures 6 and 8, respectively).

Overall, the results of this study, compared to that of [15], shows that the incorporation of vaccination with counseling and ART in the fight against HIV/AIDS will achieve faster eradication of the disease than is the case when only ART is administered.

\section{References}

[1] Anzala, O., Mutua, G. N., Oyugi, F. J. O., Mohamed, B. F., Achia, T., and Stover, J.(2012). What impact would an HIV/AIDS vaccine have on the HIV/AIDS epidemic in kenya? Open Journal of Immunology Vol. 2, No. 4, 195-201.

[2] AVERT (2010): Averting HIV/AIDS Epidemic: Worldwide HIV and AIDS Statistics Commentary.

[3] Beltrami, E. (1989): Mathematics for dynamic modeling. Academic Press. N. Y.

[4] Blower, S.M. and Mclean, A. R. (1994): Prophylactic vaccines, risk behavior change, and the probability of eradicating HIV in San Francisco. Science 265, 1451-1454.

[5] Centres for Disease Control and Prevention (CDC, 1982): Update on Acquire Immunodeficiency Syndrome (AIDS): United State-Morbidity and Mortality Weekly Report, 31, pp 507-514.

[6] Centres for Disease Control and Prevention (CDC, 1999): HIV and its transmission. Atlanta. U.S.A.

[7] Elizabeth Glaser Pediatric AIDS Foundation (EGPAF, 2013). Issue Brief: Pediatric HIV and AIDS.

[8] Garcia-Calleja, J. M., Gouws, E., and Ghys, P. D. (2006): National population based HIV prevalence survey in sub-Saharan Africa: results and implications for HIV and AIDS estimates. Sexually transmitted Infections, 82: 64-70.

[9] Greenhalgh, D., Doyle, M., and Lewis, F. (2001): A Mathematical treatment of AIDS and Condom use. IMA. J. Math. Appl. Med. Biol., 18, no.3, pp225-262.
[10] Hsieh, Y. H. (1996): A two sex model for treatment of AIDS and behaviour changes in a Population of varying size. IMA. J. Math. Appl. Biol. Med., 13, 151-173.

[11] IAVI (2011). International AIDS vaccine Initiative: "The Potential Impact of an AIDS vaccine in Low-and Middle-Income Countries"-Technical Report. New York.

[12] Kai Sun, Shuntai Zhou, Ray Y. Chen, Myron S. Cohen, and Fujie Zhang (2010): Recent Key advances in human immunodeficiency virus medicine and implications for China, AIDS Research and Therapy, 7:12.

[13] Kgosimore M. and Lungu E. M. (2004): The effects of vaccination and treatment on the spread of HIV/AIDS. Journal of Biological Systems, Vol. 12.No. 4, 399-417

[14] Kimbir, A. R. and Aboiyar, T. (2003). A mathematical model for the prevention of HIV / AIDS in a varying population. Journal of Nigerian Mathematical Society 22, 43- 55.

[15] Kimbir, A. R. and Oduwole H. K. (2008): A Mathematical Model of HIV/AIDS Transmission Dynamics Considering Counselling and Antretroviral Therapy. J. Modern Mathe. Stat., 2(5): 166-169.

[16] Koob, J. J. and Harvan J. S. (2003): AIDS instruction in US Schools of Social Work: 20 years into the epidemics. Social Work Education, 22: 309-319.

[17] Kribs-Zaleta, C. M., and Velasco-Hernandez, J. X. (2000): A simple vaccination model with multiple endemic states. Math. Biosci. 164, no. 2,183-201.

[18] Medley, G. F., Anderson, R. M., Cox, D. R., and Billard, L. (1987): Incubation period of AIDS in patients infected via bloodtransfusion. Nature, 328, pp718-724.

[19] Mugisha J.Y.T. (2005): Balancing Treatment and Prevention: The case of HIV/AIDS. American Journal of Applied Sciences 2(10): 1380-1388.

[20] Mukandavire Z., Bowa K., and Garira W. (2007): Modeling Circumcision and Condom use as HIV/AIDS preventive control strategies, Mathematical and Computer Modelling 46, 1353-1372.

[21] Putzel J. (2003): "Institutionalizing an Emergency Response: HIV/AIDS and Governance in Uganda and Senegal." Department of International Development, 5-14.

[22] Richard A. Kimbir, Martins J. I. Udoo and Terhemen Aboiyar (2012): A Mathematical Model for the Transmission Dynamics of HIV/AIDS in a two-sex Population considering Counseling and Antiretroviral Therapy (ART). J. Math. Comput. Sci. 2, No.6, 1671-1684.

[23] Swanson, C. E., Tindall, B. and Cooper, D. A. (1994): Efficacy of Zidivudine treatment in homosexual men with AIDS-related complex: factors influencing development of AIDS, survival and drug tolerance-AIDS.Vol.9, 625-634.

[24] UNAIDS (2008): Report on the global AIDS epidemic. Geneva.

[25] UNAIDS (2009): AIDS epidemic update.

[26] UNAIDS (2010): 2010 Report on global AIDS epidemic. Geneva.

[27] UNAIDS (2012): UNAIDS REPORT ON THE GLOBAL AIDS EPIDEMIC. 
[28] UNAIDS (2013): UNAIDS 2013 Global Fact Sheet.

[29] Velasco-Hernandez J. X., Gershengorn H. B., and Blower S M. (2002): Could widespread use of combination antiretroviral therapy eradicate HIV epidemics? The Lancet 2, 487-493.
[30] Williams B. G., Lloyd-Smith J. O., Gouws E., Hankins C., Getz W. M., Hargrove J., de Zoysa I., and Auvert B. (2006): The Potential Impact of Male Circumcision on HIV in sub-Saharan Africa, PLoS Medecine 3, 1032-1040. 